\title{
A gene expression profile of stem cell pluripotentiality and differentiation is conserved across diverse solid and hematopoietic cancers
}

\author{
Nathan P Palmer ${ }^{1 \dagger}$, Patrick R Schmid ${ }^{1 \dagger}$, Bonnie Berger ${ }^{1,2^{*}}$ and Isaac S Kohane ${ }^{3^{*}}$
}

\begin{abstract}
Background: Understanding the fundamental mechanisms of tumorigenesis remains one of the most pressing problems in modern biology. To this end, stem-like cells with tumor-initiating potential have become a central focus in cancer research. While the cancer stem cell hypothesis presents a compelling model of self-renewal and partial differentiation, the relationship between tumor cells and normal stem cells remains unclear.

Results: We identify, in an unbiased fashion, mRNA transcription patterns associated with pluripotent stem cells. Using this profile, we derive a quantitative measure of stem cell-like gene expression activity. We show how this 189 gene signature stratifies a variety of stem cell, malignant and normal tissue samples by their relative plasticity and state of differentiation within Concordia, a diverse gene expression database consisting of 3,209 Affymetrix HGU133+ 2.0 microarray assays. Further, the orthologous murine signature correctly orders a time course of differentiating embryonic mouse stem cells. Finally, we demonstrate how this stem-like signature serves as a proxy for tumor grade in a variety of solid tumors, including brain, breast, lung and colon.

Conclusions: This core stemness gene expression signature represents a quantitative measure of stem cellassociated transcriptional activity. Broadly, the intensity of this signature correlates to the relative level of plasticity and differentiation across all of the human tissues analyzed. The fact that the intensity of this signature is also capable of differentiating histological grade for a variety of human malignancies suggests potential therapeutic and diagnostic implications.
\end{abstract}

\section{Background}

There have been numerous investigations into the relationship between normal organogenesis programs and malignancy, particularly with respect to the stem cell properties of self-renewal and pluripotentiality [1-3]. At the molecular level, certain malignant tumors and developing tissues have been shown to exhibit shared transcription factor activity, regulation of chromatin structure, signaling characteristics and gene expression characteristics [4]. Likewise, enrichment patterns of well-characterized gene sets have been observed to be similar in stem cells and breast cancers,

\footnotetext{
* Correspondence: bab@mit.edu; isaac_kohane@hms.harvard.edu † Contributed equally

${ }^{1}$ Computer Science and Artificial Intelligence Laboratory, Massachusetts Institute of Technology, 77 Massachusetts Avenue, Cambridge MA 02139, USA

${ }^{3}$ Center for Biomedical Informatics, Harvard Medical School, 25 Shattuck Street, Boston MA 02115, USA

Full list of author information is available at the end of the article
}

bladder cancers and poorly differentiated glioblastomas [5]. In addition, a variety of stem cell populations have been identified that are specific to individual tissues, yet share some of the same gene expression characteristics of embryonic stem (ES) cells [6]. However, multiple controversies continue to circulate around the role of particular genes in stem cells versus differentiated tissues (for example, N-cadherin [7]), and the extent to which the activation of various stem cell-like programs and pathways occurs across various tissues and diseases.

The cancer stem cell hypothesis asserts a model of tumorigenesis that may tie some of these observations together [8]. By implying a hierarchical organization of tumor growth that closely reflects normal tissue development, the hypothesis simultaneously accounts for the high degree of functional heterogeneity observed in solid tumors $[9,10]$, as well as the fact that only a small fraction of malignant cells retain tumor-initiating potential [8].

\section{Biomed Central}


Under these assumptions, expression profiles derived from resected tumor samples (comprising both the cancer stem cells and their differentiated progeny) should broadly resemble those of the normal tissue of origin, with a degree of stem cell like activity also apparent.

Originally identified in hematopoietic cancers, leukemic stem cells were observed to express several markers $\left(\mathrm{CD} 34^{+} \mathrm{CD} 38^{-}\right)$in common with normal stem cells [11]. Subsequently, analogous models have been developed for a number of solid tumors, primarily through the identification of a small population (typically $<5 \%$ ) of tumor cells that were unique both in their expression of a set of specific surface markers as well as their ability to induce phenocopies of their original tumors in xenograft and transplant models [12-19].

Although the cancer stem cell model and the experimental approach to identifying cancer stem cell populations have been replicated across a variety of tissues, the molecular signatures derived from the proliferative cells have varied widely. As yet, the extent to which there exist any molecular fingerprints commonly attributable to multiple types of cancer stem cells remains unclear. While some have been observed to express a subset of the embryonic stem cell-associated genes (POU5F1, NANOG), the degree to which these trends may be broadly apparent is unknown [20].

The increasing volume of evidence supporting a pervasive connection between cancer and stem cells suggests significant therapeutic implications. As opposed to current therapies that are evaluated based on their ability to reduce the overall size of a tumor, regimens that target cancer stem cells may have more success in preventing long-term recurrence [8]. Molecular signatures that are capable of grading pluripotentiality and proliferative potential represent an important step in designing such regimens and guiding therapeutic procedures.

Indeed, gene expression signatures derived from breast cancer stem cells have been shown to separate patients with early-stage breast cancer into high-risk and low-risk groups [21]. Similarly, gene expression signatures have been used to identify cell-sorted acute myeloid leukemia (AML) samples enriched for leukemic stem cells, and leukemic stem cell expression signatures have been shown to correlate with patient survival $[22,23]$. Diverse malignant tissue samples have been shown to exhibit a broadly similar trend within a large gene expression database, but no specific connection has been made in this context to stem cell-like activity [24]. However, identifying an unbiased transcriptional measure of 'stemness' conserved across embryonic and adult stem cells, and relating that signature to malignancy, has remained a challenge $[6,25,26]$. Understanding the mechanisms of tumor proliferation and the relationship of those mechanisms to stem cell pluripotentcy may yield especially important insights into the origins and treatment of germ cell tumors, and embryonal carcinomas in particular, which have been previously demonstrated to express the hallmark ES regulators [27].

Here, we present a comprehensive analysis of a diverse compilation of gene expression samples that reveals a robust multidimensional continuum from ES/induced pluripotent stem cells to fully differentiated tissues. Our results indicate that, within this functional genomic landscape, cancers display a combination of stem cell-like programming and tissue-specific signatures. We derive a shared molecular measure of pluripotentiality that may help bridge the gap between disparate tissue-specific cancer stem cell populations, reflecting their shared proliferative potential. In addition, we demonstrate that our differentiation and pluripotentiality-centric view of gene expression correlates with classical grading systems for a variety of solid tumors, suggesting that our results may form a quantitative axis with practical relevance to personalized medicine.

\section{Results}

\section{Identifying a stem cell gene set}

Our first goal was to identify a set of genes whose expression profiles represent a tightly conserved core of transcriptional programming among stem cells. We call this set of genes the stem cell gene set (SCGS). We derived the SCGS from a high-quality database called Concordia, representing a significant subset of the NCBI's Gene Expression Omnibus (GEO) [28]. Concordia was constructed using a combination of automated textual parsing, human curation and normalization methods (see Materials and methods).

In order to identify a set of genes with highly specific stem cell expression intensities, we used this curated database to identify all of the stem cell samples in our dataset. We then applied a standard signal processing tool, a finite impulse response filter (FIR) [29], to identify those genes with the most highly conserved expression intensities among the stem cell samples. That is, those genes with a range of expression intensities among the stem cell samples that was most distinct from the nonstem cell samples scored the highest (see Materials and methods).

In contrast to a standard $t$-test, this approach does not require us to define a specific 'control' phenotype against which we test for separation, a poorly defined task when comparing against such a diverse database. Moreover, this method identifies genes with expression levels that are highly specific in the stem cell samples, allowing for the diverse population of non-stem cell samples to express these genes at simultaneously higher and lower levels (something for which a $t$-test cannot directly account). For example, the gene $D B C 1$ exhibits a highly specific range of expression across the stem cell samples, 
and ranked highly (among the top $0.5 \%$ of all genes) in its ability to localize the stem cell samples by the described method. However, the non-stem cell samples demonstrate both higher and lower expression levels of this gene (Figure 1), causing a standard Student's $t$-test (treating all non-stem cell samples as the control group) to rank this gene at only the $24.6 \%$ strongest among all genes.

We verified the ability of the SCGS to capture a nuanced measure of stem cell-like gene expression activity by demonstrating the accurate clustering of a series of developing ES cell populations in mouse (see below). This analysis also shows the concordance between the
SCGS transcriptional profile and cellular state of differentiation.

Previous studies have examined the expression patterns of literature-curated gene sets relating to ES-like activity among a variety of malignancies [5]. In contrast, we have constructed a gene set in silico that reflects only those transcriptional signals with the greatest ability to localize the stem cell samples within the spectrum of human tissues and diseases.

The 189 genes comprising the SCGS can be found in Tables s1 to s4 in Additional file 1. A variety of FIR thresholds were evaluated according to the ability of the implied gene sets to differentiate between stem cell

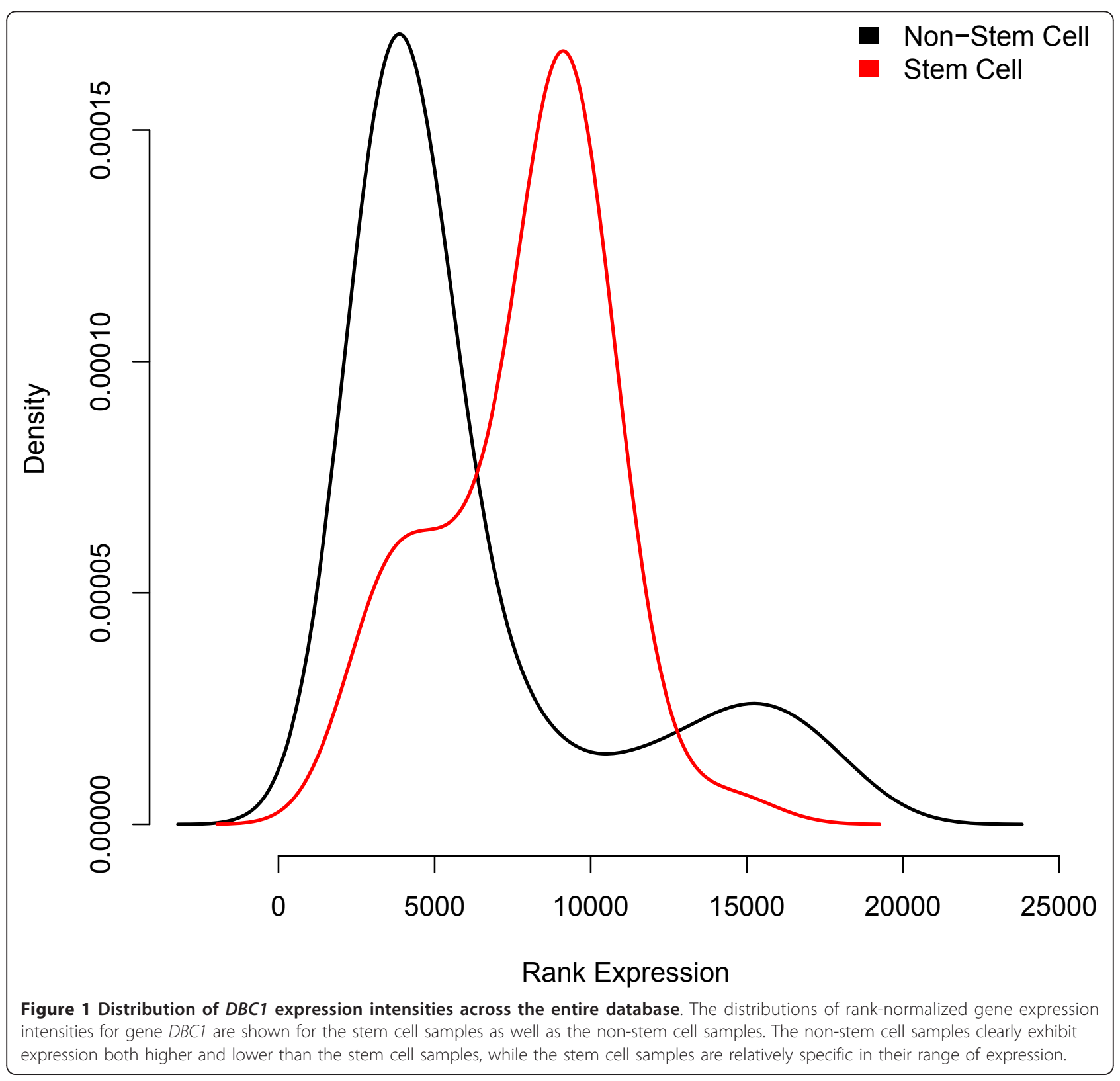


samples and the other phenotypes in the dataset via an analysis of variance (ANOVA). The genes presented here represent a set capable of simultaneously separating the pluripotent, multipotent, progenitor, malignant and normal samples, while also retaining tissue-specific features (for example, clearly separating normal blood, neural and epithelial tissues). An animation demonstrating the effect of varying the number of top-ranking stem genes included in the SCGS is included in Additional file 2.

\section{Comparison to previously published stem gene sets}

Several previous attempts have been made to identify the genes responsible for maintaining pluripotency by analyzing the expression patterns of germ cell tumors. Sperger et al. [30] performed differential expression analyses between control differentiated cells and ES cells and a variety of germ cell tumors to identify genes with higher expression in pluripotent stem cells. Our approach differs in that we analyzed the expression of only stem cells rather than cultured tumor cell lines. Further, we place no stipulation on differential expression with respect to a fixed control group, but rather focus in on the genes with the greatest ability to characterize the stem cells within a broad spectrum of the human transcriptional landscape. Skotheim et al. [31] and Almstrup et al. [32] take similar approaches, identifying the genes that characterize an assortment of germ cell tumors. Figure 2 shows the overlap of the SCGS with these previously identified stem gene sets.

\section{Stem-like signature stratifies a diverse expression database by pluripotentiality and malignancy}

Via principal component analysis (PCA), we examined the transcriptional profile of the SCGS across the entire collection of normal tissues, cancers and stem cells assembled from GEO. Performing PCA across only the SCGS genes (including all samples in the data set) allowed us to measure the extent to which the specific transcriptional activity observed in the stem cell population was apparent in each of the other phenotypes.

This analysis revealed a striking trend apparent in the first two principal components (PCs) of the gene set; most importantly, $\mathrm{PC} 1$ captured a measure of cellular pluripotency, while PC2 reflected the broad transcriptional differences between hematopoietic, neural and epithelial tissues. These trends are demonstrated in Figure 3. Each panel highlights in color the PCA region occupied by a particular normal tissue population (red) and its associated malignancies (green), as well as any related precursor cells (orange), immortalized cell line samples (cyan), multipotent (blue) and pluripotent stem cells (magenta) (PCA was computed jointly across all samples; each cancer is highlighted individually for clarity). The pluripotent stem cells included in this analysis were a combination of both ES cells and induced pluripotent stem cells. The locations of all other samples in the data set are shaded gray to provide context.

The dominant characteristic of PC1 is its ability to separate the pluripotent stem cells from the normal tissue samples (for example, the normal tissues shown in Figure 3 - blood, breast, brain, colon, shaded red, consistently lie on the extreme left side of the plots, whereas the pluripotent stem cells, shaded magenta, lie on the extreme right). Moreover, PC1 apparently reflects a finer-grained continuum of cellular potency: the multipotent stem cells are clustered near the pluripotent stem cells, with the hematopoietic progenitors (the only progenitors in our dataset) slightly farther away (Figure 3a).

Further, the hematopoietic, neural and epithelial cancers (shaded green in Figure 3a-d) contained in our data all clustered directly between the stem cell populations and their associated normal non-malignant samples. This suggests that the SCGS captures a kernel of stem cell-like transcriptional activity that is concurrently apparent in a variety of malignancies. These findings build on previous observations that genes associated with stem cell-like activity demonstrate differential expression in a variety of epithelial cancers with respect to their normal tissue counterparts [6]. Our analysis reveals that stem-like expression profiles are observable not only in epithelial cancers, but also in neural and hematopoietic malignancy as well.

We will use the coordinates of an expression profile's projection into the first principal component of the gene space defined by the SCGS as a relative measure of 'stemness', our stemness index.

The overall landscape of the human transcriptome appears to be organized by a combination of tissue, celltype and disease-specific features [24]. Previous studies have suggested that the primary factors driving the organization of this landscape are largely attributable to hematopoietic and malignant programming [24]. Our results indicate that while there exists a strong tissue-specific signal, the 'malignancy' signature is more specifically a reflection of the self-renewal and pluripotentiality common to both stem cell populations and heterogeneous tumors.

\section{Human-derived ES-like transcriptional profile correlates to mouse stem cell differentiation}

To verify that our SCGS-derived stemness index captures a quantitative transcriptional measure of differentiation, we used it to examine the expression dynamics of a set of developing mouse ES cells over time [GEO: GSE12550]. This data set consisted of a time-course of differentiating mouse ES cells, with gene expression measured at four time points (ES cells, 4 days of differentiation, 8 days of differentiation and 14 days of differentiation). 


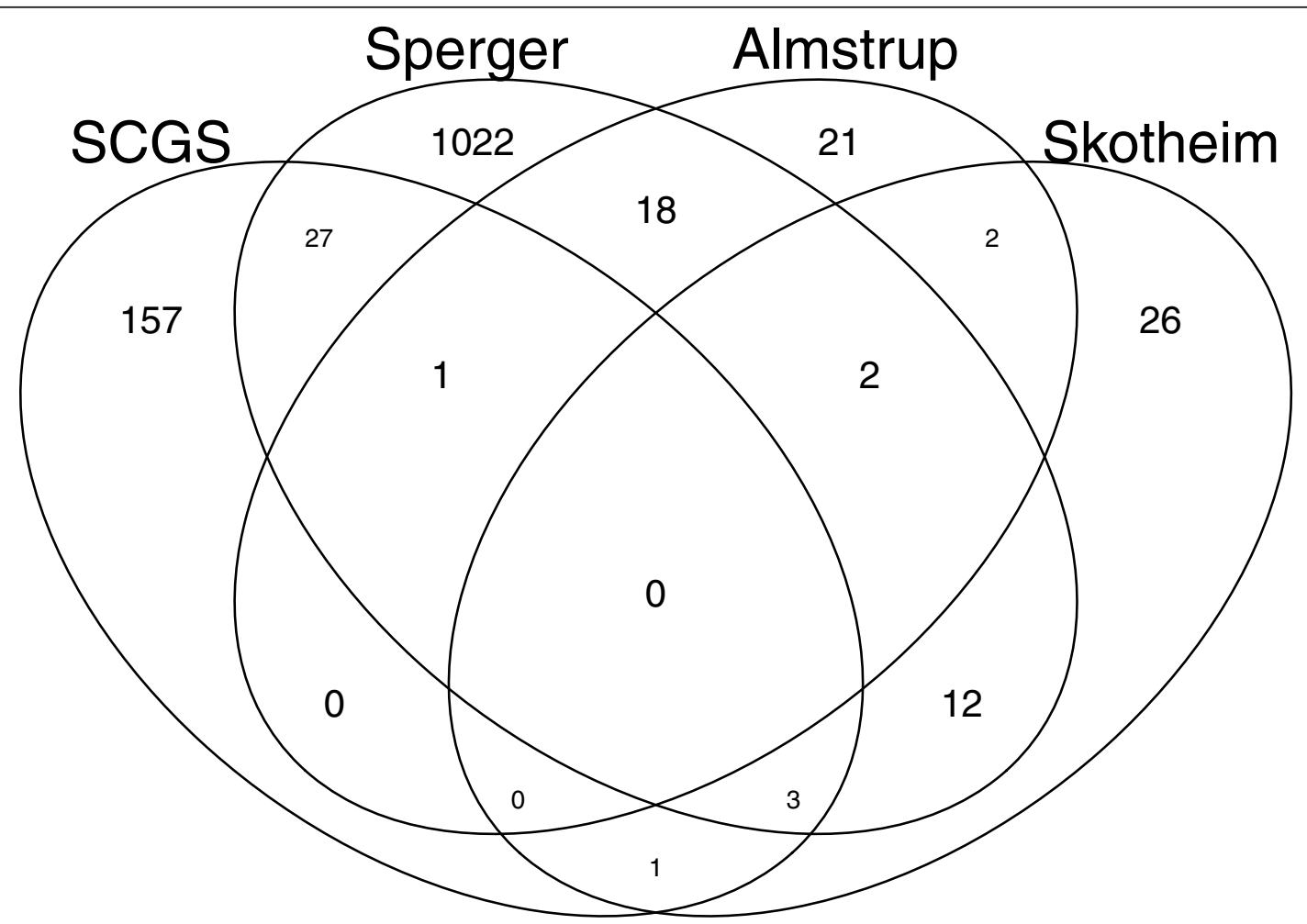

Figure 2 SCGS overlap with previously identified stem cell genes. The Venn diagram shows the number of genes in common and distinct to each of the gene sets: Sperger et al. [30]; Skotheim et al. [31]; Almstrup et al. [32].

Human SCGS gene IDs were mapped to mouse via NCBI's HomoloGene [33]. Human genes that lacked a unique match in mouse were ignored. Expression intensities were processed in an identical manner to the human data (see Materials and methods) and summarized by gene. Again, we computed the dominant variance among the differentiating mouse cells via PCA over the SCGS. We likewise used each mouse ES sample's stemness index (that is, coordinates in the first principal basis) as a summary value of SCGS gene expression activity.

The dominant expression signal reflected in these genes accurately sorts the samples according to their time point, as shown in Figure 4. This supports the hypothesis that our SCGS-derived stemness index reflects measurable changes in state of differentiation and pluripotentiality, and reflects the fact that the functional genomic mechanisms associated with stem cell activity are at least partially conserved across species [34].

\section{Stratifying tumor grade}

We used the stemness index that we derived from the SCGS to evaluate the transcriptional profiles of several graded tumor data sets. Our goal was to evaluate whether our molecular marker for tissue-agnostic stem cell-like transcriptional activity was representative of poor clinical prognosis. We included four publicly available data sets in this analysis (see Materials and methods). For each data set, we computed the samples' stemness index (via PCA over the SCGS) to identify the dominant differences between the samples within the context of the stem cell genes (Materials and methods).

This analysis revealed that our stemeness index correlates with tumor grade for a variety of primary tissues. Figure 5 shows the distribution of stemness index values for the four tissue types' graded tumor samples. In each case, the transcriptional activity of the SCGS defines a clear separation between the high- and low-graded tumors, while also providing a molecular foundation based on stem-like expression for the clinical difficulty in classifying mid-grade tumors $[35,36]$. Importantly, such measures should not be considered in isolation, but in concert with standard histopathology, since an aggressive tumor containing a relatively large proportion of normal cells would likely have a low stemness score. As such, these methods may well serve as a 'warning sign' when traditional pathology assigns a low grade, but RNA analysis suggests the tumor is about to turn aggressive.

Recent trends in chemotherapy design have focused not only on regulating cytotoxicity, but also on affecting the differentiation pathways that are apparently impaired in malignant cells. For example, Stegmaier et al. [37] have demonstrated the ability of gefitinib to induce myeloid 


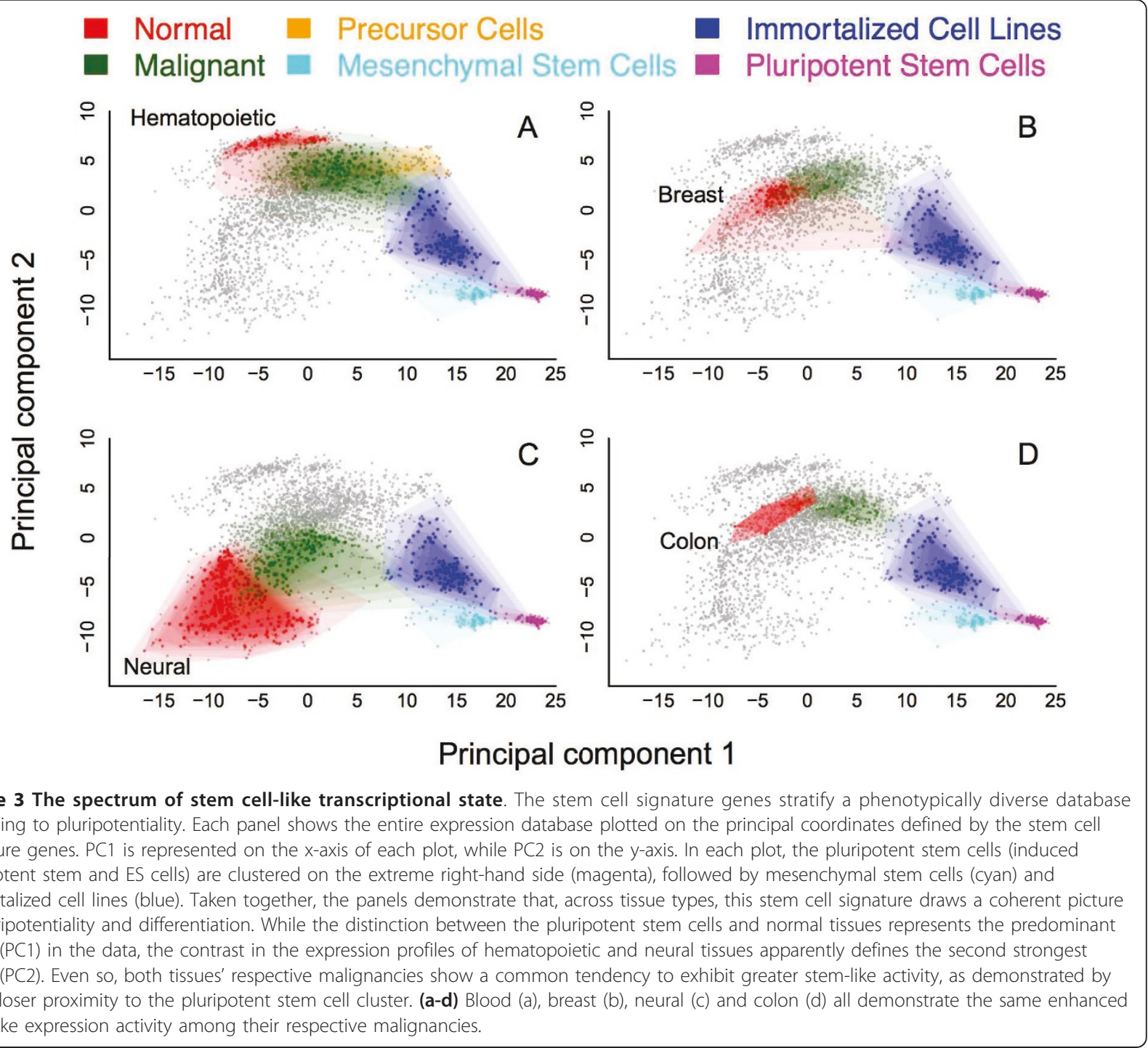

differentiation in both acute myeloid leukemia cell lines as well as patient-derived acute myeloid leukemia blast cells. Indeed, the phenotypic transformation induced by gefitinib was shown to be observable in both cellular morphology and gene expression. The ubiquitous stem cell-like expression patterns described in this paper, as well as those specifically tuned to individual tumor subclasses, may prove useful in screening compounds through the early stages of drug discovery. Understanding the transcriptional changes wrought by these compounds within the context of pluripotentiality and differentiation may be of fundamental value in personalized oncology and therapy selection.

\section{Functional diversity of the stem cell gene set}

Our final goal was to characterize the functional diversity of the genes comprising the SCGS. Hierarchical clustering of these genes' transcriptional activity in a population of pluripotent stem cells revealed four distinct coexpression modules. For each module, we then identified a set of over-enriched Gene Ontology (GO) biological processes [38].

To illustrate the gene expression trends apparent within each gene cluster, Figure 6 shows a heatmap of their profiles across pluripotent and partially committed stem cells, as well as malignant and normal breast samples. Genes active in DNA replication, cell cycle regulation and RNA transcription (see Tables s5 and s6 in Additional file 1 for detailed annotations) are most highly expressed in the pluripotent stem cells, and less so, respectively, through increasing levels of cellular differentiation/decreasing pluripotentiality, consistent with prior studies of the dynamics of stem cell cycling and regeneration [25,39]. Genes related to metabolism and hormone signaling (Table s7 in 


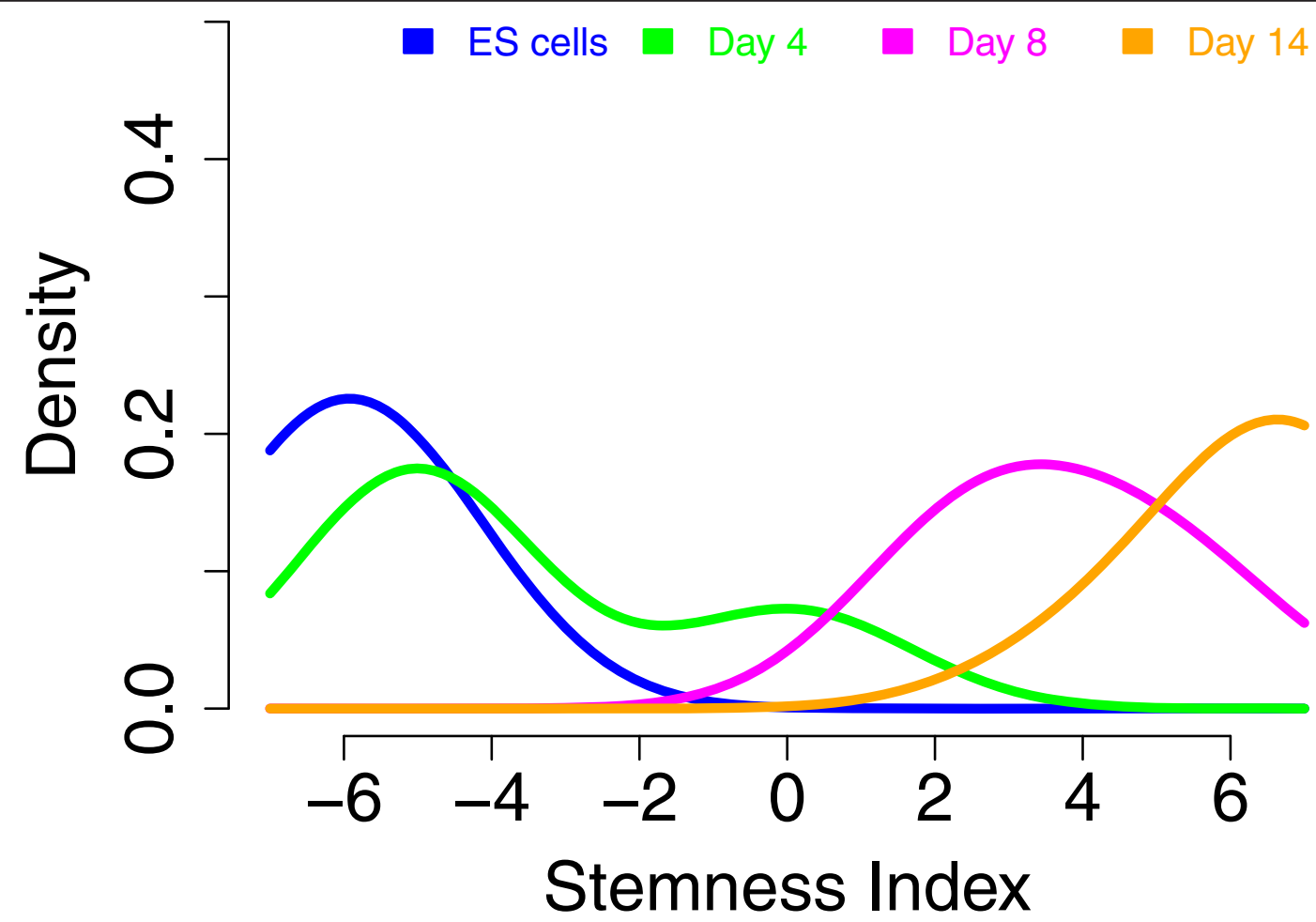

Figure 4 Distribution of differentiating mouse ES cells over stemness index. Each curve represents the distribution of stemness index values for a particular time point. This signature collocates the four time points' samples and clearly separates the early and late stages of differentiation.

Additional file 1) show peak expression intensity among the partially committed stem cells, while exhibiting low intensity among the fully differentiated tissue and tumor samples. Correspondingly, genes responsible for multicellular signaling and cellular identity (Table s8 in Additional file 1) are most highly expressed in the fully differentiated tissue and malignant samples. Within each functional module, the tumor samples trend away from the respective normal tissue, echoing stem cell-like transcriptional activity.

\section{Conclusions}

We have demonstrated conserved stem cell-like transcriptional activity across a wide variety of hematopoietic and solid cancers through a comprehensive molecular survey of malignancy, pluripotent stem cells and normal tissues. Our findings echo several recent developments in the cancer stem cell debate. In particular, our results highlight transcriptional evidence that, despite individual tissue-specific characteristics, a wide range of cancers share a common set of transcriptional mechanisms with each other, as well as pluripotent and multipotent stem cells.

While a large volume of evidence indicates that only a small number of tumor cells are capable of self-renewal, controversy remains as to the exact origin of these cells. The hierarchical cancer stem cell hypothesis suggests that these cells arise from normal pluripotent or multipotent stem cells that have lost the ability to regulate their proliferative activity. Under this model, the phenotypic diversity observed in many tumors is viewed as the result of this defective stem cell population mismanaging the process of normal organogenesis. Alternatively, the stochastic model of tumorigenesis suggests that proliferative tumor cells arise from normal fully differentiated or committed progenitor cells that acquire the ability to self renew, and that tumor cell phenotype variation is the result of these mutated cells differentiating in a random fashion [40].

Regardless of the origin of proliferative tumor cells, our results indicate that there is a high degree of stem cellspecific gene expression programming observable in heterogeneous tumor samples. Our data indicate the need for more detailed transcriptional assays comparing proliferative tumor cells to both ES/induced pluripotent stem cells and bulk heterogeneous tumor cells, as well as normal tissue cells. Our data suggest the hypothesis that the gene expression patterns observed in heterogeneous tumor samples may be due to the effect of a small population of cancer stem cells in combination with a large 


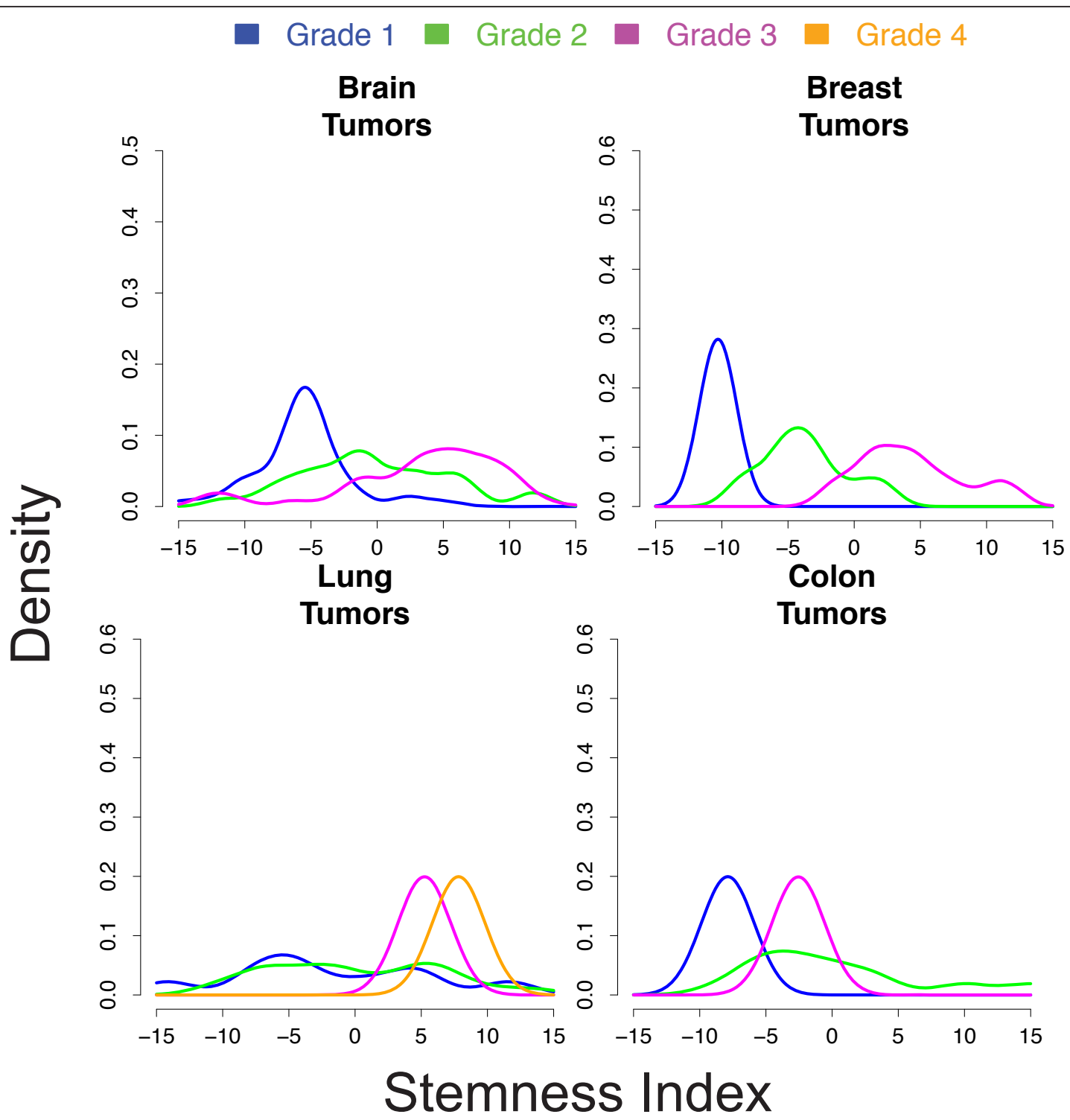

Figure 5 Stem cell-like activity correlates with tumor grade in various solid malignancies. Each panel displays the distribution, within the space of the stem cell genes, of graded tumor samples for one particular tissue type. Our stemness index consistently separates high-grade tumors from low grade ones. Based on this transcriptional index, the mid-grade tumors are less well defined.

number of partially differentiated cells. It is plausible that, while the partially differentiated mass of the tumor behaves transcriptionally similarly to healthy tissue, the small population of proliferative tumor cells pushes the observation of the aggregate mRNA back along the spectrum of stem cell-like activity identified in this paper.

We have demonstrated a specific transcriptional signal that is shared among a wide variety of solid and hematopoietic cancers. Moreover, when considered from a transcriptome-wide perspective, this signal is indicative of stem cell-like activity. We have shown how these gene expression patterns are most strongly associated with embryonic and induced pluripotent stem cells, and are successively less apparent in multipotent stem cells, malignancies, and fully differentiated tissues, respectively. In addition, the genes that comprise this signal also reveal a stratification of solid tumors that correlates strongly with classical grading systems.

\section{Materials and methods}

Concordia, a large phenotypically diverse gene expression database

The Concordia database contains 3,209 Affymetrix HGU133+ 2.0 gene expression array samples (all from human tissue or cultured human cell lines) extracted from NCBI's GEO. A full description of the techniques 


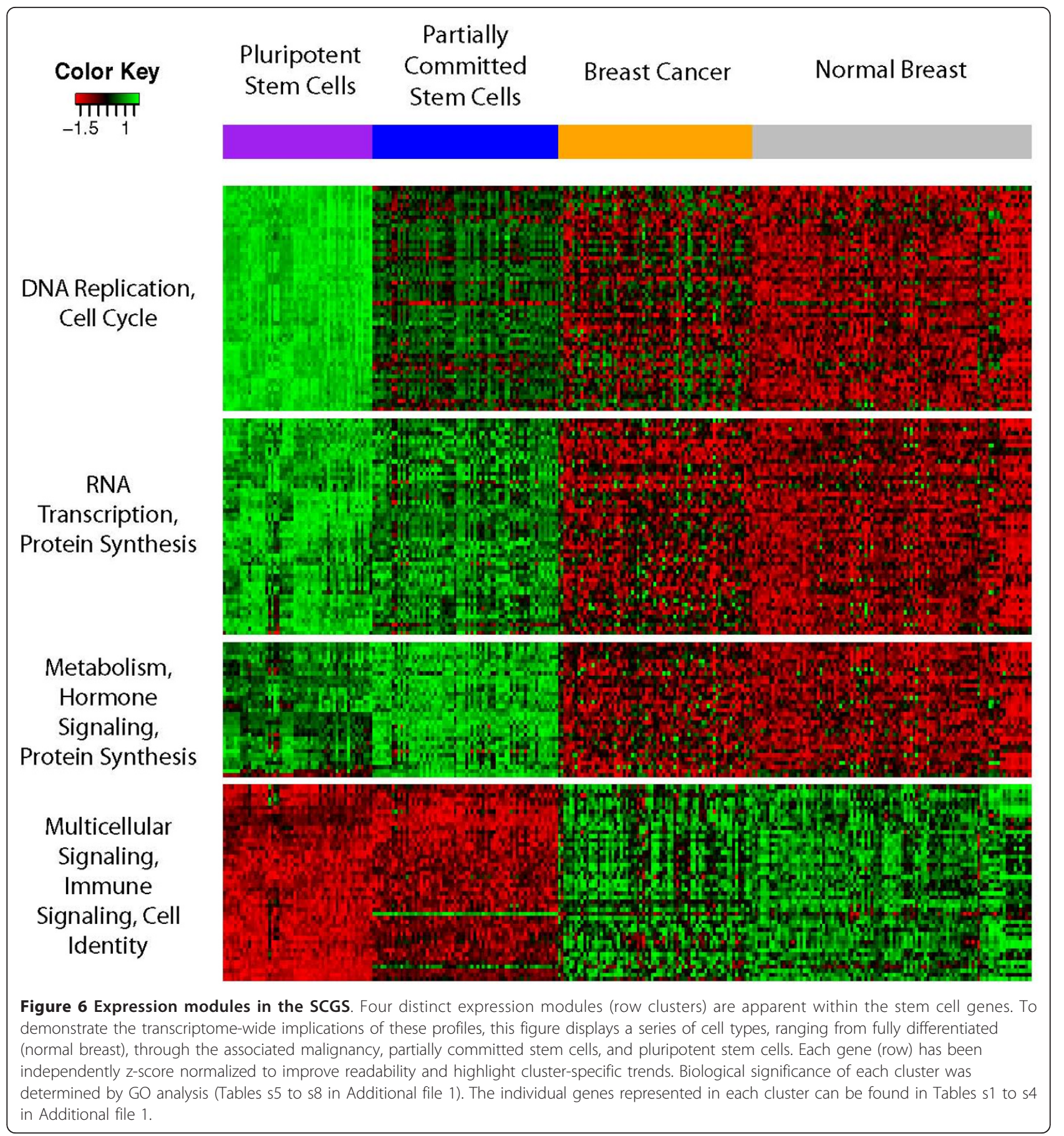

used to assemble this database have been previously described [41], and the curated phenotype data are available for public download at the Concordia database web site [42], including all of the non-malignant, malignant and stem cell samples, less the external graded tumor sets that were used to verify the SCGS signal's relationship to solid tumor histology. The following two sections briefly describe the Concordia database.
Using UMLS annotation to associate each sample with its relevant phenotypes

We constructed a database representing a subset $(3,209$ samples) of NCBI's GEO $[28,33]$ that contained a combination of samples derived from normal tissues, immortalized cell lines, a variety of cancers, and an assortment of pluripotent and partially committed stem cells. In order to generate high-quality, systematic 
phenotype annotations for this dataset, the GEO text descriptions relating to each sample (including title, description, and source fields) were mapped into the Unified Medical Language System's (UMLS) [43] ontology of biological and medical concepts. This was done using a combination of natural language processing (NLP) software and hand validation to remove spurious associations.

NLP was performed by the Java implementation of the National Library of Medicine's (NLM) MetaMap program, MMTx [44]. A custom UMLS thesaurus was generated using NLM's MetaMorphosys program that contained the concepts and relationships from the UMLS, MeSH, and SNOMED ontologies.

These automated annotations were then verified by hand so as to remove false positives. Using custom-built software, these associations were propagated through the ontology's hierarchy, allowing us to identify all samples related to phenotypes of arbitrary specificity.

\section{Normalizing the gene expression samples}

The expression data for the samples in our dataset were obtained from their respective GEO CEL files, which were MAS 5.0 [45] normalized via R's BioConductor package $[46,47]$. The resulting probe set intensities were averaged into 20,252 unique gene-centric values, and then rank normalized to improve cross-data series comparability. All calculations were performed in the $\mathrm{R}$ statistical environment, employing the BioConductors suite.

\section{Additional expression data}

In addition to the Concordia gene expression data, several additional GEO data sets were used to analyze the SCGS signal's relationship to histological tumor grade. These are: a series of graded glioma tumor samples [GEO: GSE4290]; a series of graded tumor samples from core needle biopsies of breast cancer patients, including a variety of estrogen receptor-positive and -negative and progesterone receptor-positive and -negative phenotypes [GEO: GSE23593]; a set of graded lung tumors, including a variety of squamous and adenocarcinoma samples [GEO: GSE18842]; and a set of graded colon tumors [GEO: GSE17537].

\section{Using FIR to identify genes that characterize pluripotent stem cells \\ We sought to associate with each gene a measure of how well conserved its expression intensity was over the stem cell samples. Rather than seeking a strict measure of con- stitutive over- or under-expression of the gene among the stem cell population, our goal was instead to identify indi- vidual genes that tightly cluster the stem cell population anywhere along the spectrum of expression intensities.}

Here, we employ a signal-processing tool, the FIR [29]. The input to this procedure is a list of all of the expression samples, sorted according to their intensity for a particular gene. The filter then applies a 'sliding window' to the list and outputs, at each window position, the proportion of stem cell samples within the frame. The maximal value of this sliding window at any position in the list is then taken as that gene's score. We use a window equal in size to the total number of stem cell samples in the database, so the interpretation of the filter's maximal output is intuitive: if we are looking to find all of the stem cell samples within one window frame, what is the greatest fraction that we can localize, given the expression values for this gene across the entire database? Genes with the highest scores are those with most specific stem cell expression intensities.

Binomial $P$-values $(k=$ number of stem cell samples in a given window frame; $n=$ window frame size; $p=$ proportion of stem cell samples in the entire database) are reported along with these scores.

To ensure that the method was not simply selecting genes that are all highly correlated with each other across the entire database, we computed the distribution of SCGS Pearson correlation coefficients over the stem cell samples, malignant tissue samples and non-malignant tissue samples independently, then compared those distributions to 1,000 random sets of genes equal in size to the SCGS. Only the non-malignant tissue samples show a positive location shift (Figure 7).

\section{Summarizing expression signals across a group of genes via PCA}

In order to capture a continuous measure of SCGS activity, we applied PCA [48]. The basis vector associated with the largest eigenvalue of the gene-gene covariance matrix captures the dominant coordinated signal present within the gene set. By projecting each sample's observed expression intensity onto this basis, we compute a summary value describing the sample's affinity for a stem cell-like gene expression profile.

\section{Measuring tumor grade along the continuum of stem-like expression}

We identified four independent data series containing expression profiles for graded tumors of various tissue types in GEO ([GEO: GSE4290], [GEO: GSE23593], [GEO: GSE17537], [GEO: GSE18842]) on Affymetrix HGU 133+ 2.0. Each series was pre-processed (MAS5.0 normalized, summarized) as previously described. Within each series, the SCGS summary values were computed, again, via PCA over this gene set, allowing us to associate a value with each sample indicating its relative stem-like expression activity. 


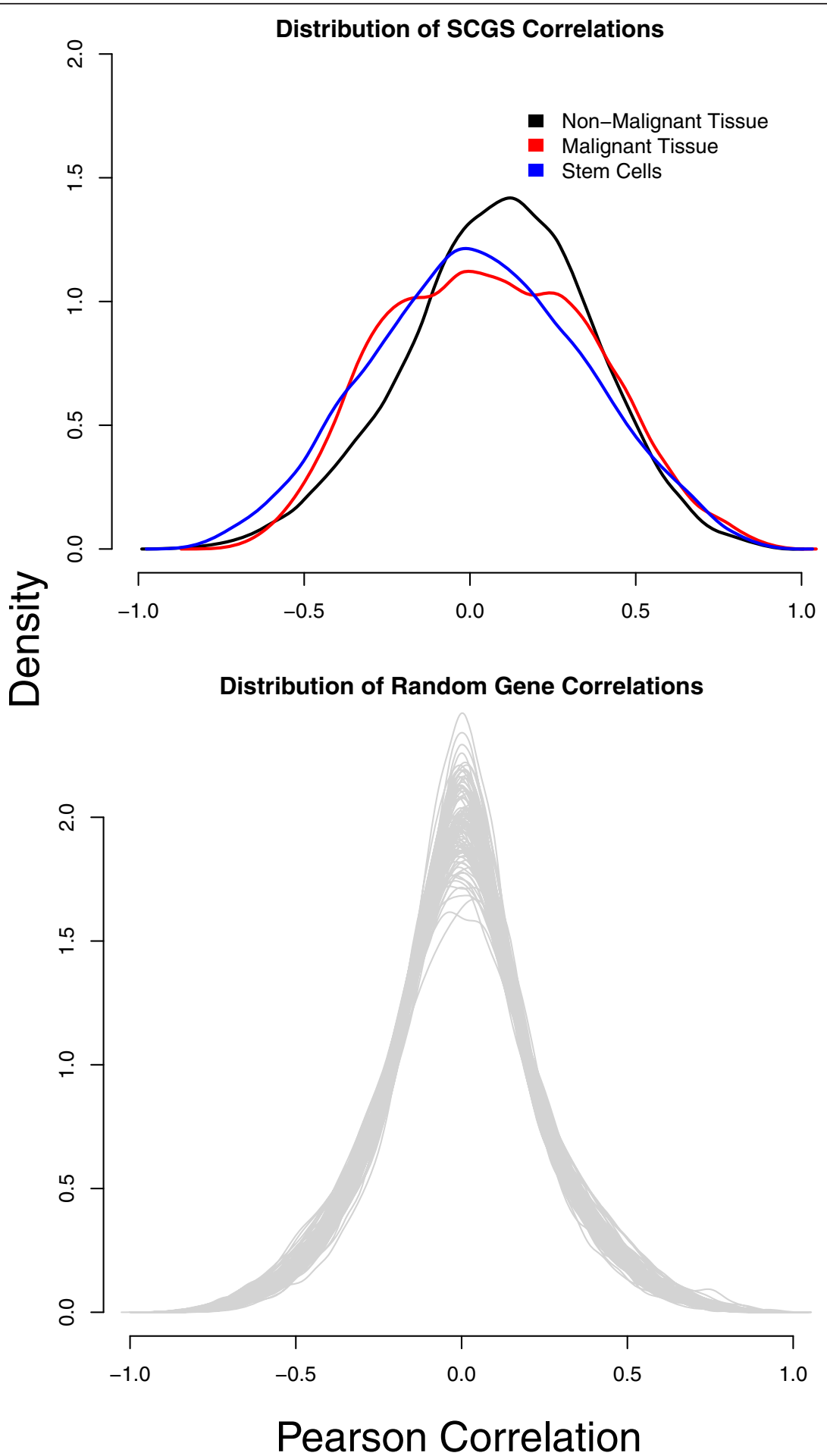

Figure 7 Inter-gene SCGS correlation across various sample types. The distribution of SCGS gene-gene correlations are shown in the top panel independently for the non-malignant, malignant and stem cell samples contained in the database. The distribution of gene-gene correlations for 1,000 random sets of genes equal in size to the SCGS is shown in the bottom panel.

SCGS clustering and GO enrichment

The SCGS was clustered using the gplots package for R. Genes were individually quantile normalized to improve readability of the resulting figures. GO biological process enrichment calculations were performed on the individual clusters using the GOstats BioConductor library [38,49]. 


\section{Data access}

All microarray samples included in these analyses are publicly available via the GEO. Accession IDs for each sample are included in Additional file 1, and curated, machine-readable phenotype information for those samples is available at the Concordia database web site [42].

\section{Additional material}

Additional file 1: Supplementary tables. Tables $s 1$ to $s 4$ : genes in the SCGS, organized by the functional module to which they belong. Tables s5 to s8: $\mathrm{GO}$ enrichment statistics for each functional module in the SCGS. The file also includes a complete listing of all of the GEO sample identifiers for the microarray data comprising our database.

Additional file 2: This file contains an animation demonstrating the effect of varying the FIR score threshold for including genes in the SCGS. For each possible number of top-scoring stem genes from 3-502 (displayed at the top of the animation frame), we project all of the samples in the database into the first two PCs of gene space (panel on top right), and highlight in color six relevant phenotypes (as in Figure 3): embryonic/induced pluripotent stem cells in magenta; mesenchymal stem cells in cyan; immortalized cell line samples in blue; blood precursor cells in orange; leukemia samples in green; normal blood in red. The panel below the PCA scatter plot shows the distribution of stemness index values (PC1 projection coordinates) for each highlighted phenotype. The plot on the left of the frame shows the analysis of variance (ANOVA) score (including all highlighted phenotypes) for the clustering defined by the current stemness index highlighted by a magenta dot on the curve showing all ANOVA scores for all of the depicted FIR thresholds. Higher ANOVA scores indicate better multi-way separation of the individual phenotypes along the stemness index. ANOVA was calculated and all plots were generated in the R statistical environment $[46,47]$.

\section{Abbreviations}

ANOVA: Analysis of Variance; ES, embryonic stem; FIR: finite impulse response filter; GEO: Gene Expression Omnibus; GO: Gene Ontology; NLM: National Library of Medicine; NLP: natural language processing; PC: principal component; PCA: principal component analysis; SCGS: stem cell gene set; UMLS: Unified Medical Language System.

\section{Acknowledgements}

The project was supported by US National Institute of Heath $(\mathrm{NIH})$ grants R01GM081871, R01LM010125-02, and U54-LM008748.

\section{Author details}

${ }^{1}$ Computer Science and Artificial Intelligence Laboratory, Massachusetts Institute of Technology, 77 Massachusetts Avenue, Cambridge MA 02139, USA. ${ }^{2}$ Department of Mathematics, Massachusetts Institute of Technology, 77 Massachusetts Avenue, Cambridge MA 02139, USA. ${ }^{3}$ Center for Biomedical Informatics, Harvard Medical School, 25 Shattuck Street, Boston MA 02115, USA.

\section{Authors' contributions}

NP contributed to the study design, contributed to data set curation, performed statistical analyses, and wrote the manuscript. PS assisted in data curation, study design and manuscript preparation. IK and BB lead the study design, advised on statistical analyses, and assisted in manuscript preparation. All authors have read and approved the manuscript for publication.

\section{Competing interests}

The authors declare that they have no competing interests.

Received: 14 March 2012 Revised: 3 August 2012

Accepted: 21 August 2012 Published: 21 August 2012

\section{References}

1. Rivera MN, Haber DA: Wilms' tumour: connecting tumorigenesis and organ development in the kidney. Nat Rev Cancer 2005, 5:699-712.

2. Scotting PJ, Walker DA, Perilongo G: Childhood solid tumours: a developmental disorder. Nat Rev Cancer 2005, 5:481-488.

3. Stiewe T: The p53 family in differentiation and tumorigenesis. Nat Rev Cancer 2007, 7:165-168.

4. Naxerova K, Bult CJ, Peaston A, Fancher K, Knowles BB, Kasif S, Kohane IS: Analysis of gene expression in a developmental context emphasizes distinct biological leitmotifs in human cancers. Genome Biol 2008, 9:R108.

5. Ben-Porath I, Thomson MW, Carey VJ, Ge R, Bell GW, Regev A, Weinberg RA: An embryonic stem cell-like gene expression signature in poorly differentiated aggressive human tumors. Nat Genet 2008, 40:499-507.

6. Wong DJ, Liu H, Ridky TW, Cassarino D, Segal E, Chang HY: Module map of stem cell genes guides creation of epithelial cancer stem cells. Cell Stem Cell 2008, 2:333-344

7. Li P, Zon LI: Resolving the controversy about N-cadherin and hematopoietic stem cells. Cell Stem Cell 2010, 6:199-202.

8. Visvader JE, Lindeman GJ: Cancer stem cells in solid tumours: accumulating evidence and unresolved questions. Nat Rev Cancer 2008, 8:755-768.

9. Heppner $\mathrm{GH}$, Miller BE: Tumor heterogeneity: biological implications and therapeutic consequences. Cancer Metastasis Rev 1983, 2:5-23.

10. Dontu G, Al-Hajj M, Abdallah WM, Clarke MF, Wicha MS: Stem cells in normal breast development and breast cancer. Cell Prolif 2003, 36(Suppl 1):59-72.

11. Fialkow PJ: Stem cell origin of human myeloid blood cell neoplasms. Verhandlungen der Deutschen Gesellschaft für Pathologie 1990, 74:43-47.

12. Singh SK, Clarke ID, Terasaki M, Bonn VE, Hawkins C, Squire J, Dirks PB: Identification of a cancer stem cell in human brain tumors. Cancer Res 2003, 63:5821-5828.

13. Al-Hajj M, Wicha MS, Benito-Hernandez A, Morrison SJ, Clarke MF: Prospective identification of tumorigenic breast cancer cells. Proc Natl Acad Sci USA 2003, 100:3983-3988.

14. Fang D, Nguyen TK, Leishear K, Finko R, Kulp AN, Hotz S, Van Belle PA, Xu X, Elder DE, Herlyn M: A tumorigenic subpopulation with stem cell properties in melanomas. Cancer Res 2005, 65:9328-9337.

15. Bapat SA, Mali AM, Koppikar CB, Kurrey NK: Stem and progenitor-like cells contribute to the aggressive behavior of human epithelial ovarian cancer. Cancer Res 2005, 65:3025-3029.

16. Collins AT, Berry PA, Hyde C, Stower MJ, Maitland NJ: Prospective identification of tumorigenic prostate cancer stem cells. Cancer Res 2005, 65:10946-10951.

17. Gibbs CP, Kukekov VG, Reith JD, Tchigrinova O, Suslov ON, Scott EW, Ghivizzani SC, Ignatova TN, Steindler DA: Stem-like cells in bone sarcomas: implications for tumorigenesis. Neoplasia 2005, 7:967-976.

18. Ricci-Vitiani L, Lombardi DG, Pilozzi E, Biffoni M, Todaro M, Peschle C, De Maria R: Identification and expansion of human colon-cancer-initiating cells. Nature 2007, 445:111-115

19. Lobo NA, Shimono Y, Qian D, Clarke MF: The biology of cancer stem cells. Annu Rev Cell Dev Biol 2007, 23:675-699.

20. Yu J, Vodyanik MA, Smuga-Otto K, Antosiewicz-Bourget J, Frane JL, Tian S, Nie J, Jonsdottir GA, Ruotti V, Stewart R, Slukvin II, Thomson JA: Induced pluripotent stem cell lines derived from human somatic cells. Science 2007, 318:1917-1920.

21. Liu R, Wang X, Chen GY, Dalerba P, Gurney A, Hoey T, Sherlock G, Lewicki J, Shedden K, Clarke MF: The prognostic role of a gene signature from tumorigenic breast-cancer cells. N Engl J Med 2007, 356:217-226.

22. Gentles AJ, Plevritis SK, Majeti R, Alizadeh AA: Association of a leukemic stem cell gene expression signature with clinical outcomes in acute myeloid leukemia. JAMA 2010, 304:2706-2715.

23. Eppert K, Takenaka K, Lechman ER, Waldron L, Nilsson B, van Galen P, Metzeler KH, Poeppl A, Ling V, Beyene J, Canty AJ, Danska JS, Bohlander SK, Buske C, Minden MD, Golub TR, Jurisica I, Ebert BL, Dick JE: Stem cell gene expression programs influence clinical outcome in human leukemia. Nat Med 2011, 17:1086-1093.

24. Lukk M, Kapushesky M, Nikkilä J, Parkinson H, Goncalves A, Huber W, Ukkonen E, Brazma A: A global map of human gene expression. Nat Biotechnol 2010, 28:322-324.

25. Ramalho-Santos M, Yoon S, Matsuzaki Y, Mulligan RC, Melton DA: "Stemness": transcriptional profiling of embryonic and adult stem cells. Science 2002, 298:597-600. 
26. Fortunel NO, Otu HH, Ng H-H, Chen J, Mu X, Chevassut T, Li X, Joseph M, Bailey C, Hatzfeld JA, Hatzfeld A, Usta F, Vega VB, Long PM, Libermann TA, Lim B: Comment on "'Stemness': transcriptional profiling of embryonic and adult stem cells" and "a stem cell molecular signature". Science 2003, 302:393, author reply 393.

27. Gillis AJM, Stoop H, Biermann K, van Gurp RJHLM, Swartzman E, Cribbes S, Ferlinz A, Shannon M, Oosterhuis JW, Looijenga LHJ: Expression and interdependencies of pluripotency factors LIN28, OCT3/4, NANOG and SOX2 in human testicular germ cells and tumours of the testis. Int J Androl 2011, 34:e160-74.

28. Barrett T, Troup DB, Wilhite SE, Ledoux P, Evangelista C, Kim IF, Tomashevsky M, Marshall KA, Phillippy KH, Sherman PM, Muertter RN, Holko M, Ayanbule O, Yefanov A, Soboleva A: NCBI GEO: archive for functional genomics data sets-10 years on. Nucleic Acids Res 2011, 39: D1005-1010.

29. McClellan JH, Schafer RW, Yoder MA: DSP First: a Multimedia Approach Prentice Hall; 1998.

30. Sperger JM, Chen X, Draper JS, Antosiewicz JE, Chon CH, Jones SB, Brooks JD, Andrews PW, Brown PO, Thomson JA: Gene expression patterns in human embryonic stem cells and human pluripotent germ cell tumors. Proc Natl Acad Sci USA 2003, 100:13350-13355.

31. Skotheim RI, Lind GE, Monni O, Nesland JM, Abeler VM, Fosså SD, Duale N, Brunborg G, Kallioniemi O, Andrews PW, Lothe RA: Differentiation of human embryonal carcinomas in vitro and in vivo reveals expression profiles relevant to normal development. Cancer Res 2005, 65:5588-5598,

32. Almstrup K, Hoei-Hansen CE, Wirkner U, Blake J, Schwager C, Ansorge W, Nielsen JE, Skakkebaek NE, Rajpert-De Meyts E, Leffers H: Embryonic stem cell-like features of testicular carcinoma in situ revealed by genomewide gene expression profiling. Cancer Res 2004, 64:4736-4743.

33. Sayers EW, Barrett T, Benson DA, Bolton E, Bryant SH, Canese K, Chetvernin V, Church DM, DiCuccio M, Federhen S, Feolo M, Fingerman IM, Geer LY, Helmberg W, Kapustin Y, Landsman D, Lipman DJ, Lu Z, Madden TL, Madej T, Maglott DR, Marchler-Bauer A, Miller V, Mizrachi I, Ostell J, Panchenko A, Phan L, Pruitt KD, Schuler GD, Sequeira E, et al: Database resources of the National Center for Biotechnology Information. Nucleic Acids Res 2011, 39:D38-51.

34. Cai J, Xie D, Fan Z, Chipperfield H, Marden J, Wong WH, Zhong S: Modeling co-expression across species for complex traits: insights to the difference of human and mouse embryonic stem cells. PLoS Comp Biol 2010, 6:e1000707.

35. Tonn JC, Westphal M: Neuro-oncology of CNS tumors Springer Verlag; 2006

36. Fuller GN, Mircean C, Tabus I, Taylor E, Sawaya R, Bruner JM, Shmulevich I, Zhang W: Molecular voting for glioma classification reflecting heterogeneity in the continuum of cancer progression. Oncol Rep 2005, 14:651-656.

37. Stegmaier K, Corsello SM, Ross KN, Wong JS, Deangelo DJ, Golub TR: Gefitinib induces myeloid differentiation of acute myeloid leukemia. Blood 2005, 106:2841-2848.

38. Ashburner M, Ball CA, Blake JA, Botstein D, Butler H, Cherry JM, Davis AP, Dolinski K, Dwight SS, Eppig JT, Harris MA, Hill DP, Issel-Tarver L, Kasarskis A, Lewis S, Matese JC, Richardson JE, Ringwald M, Rubin GM, Sherlock G: Gene ontology: tool for the unification of biology. The Gene Ontology Consortium. Nat Genet 2000, 25:25-29.

39. Takizawa H, Regoes RR, Boddupalli CS, Bonhoeffer S, Manz MG: Dynamic variation in cycling of hematopoietic stem cells in steady state and inflammation. J Exp Med 2011, 208:273-284.

40. Gupta PB, Fillmore CM, Jiang G, Shapira SD, Tao K, Kuperwasser C, Lander ES: Stochastic state transitions give rise to phenotypic equilibrium in populations of cancer cells. Cell 2011, 146:633-644.

41. Schmid PR, Palmer NP, Kohane IS, Berger B: Making sense out of massive data by going beyond differential expression. Proc Natl Acad Sci USA 2012, 109:5594-5599.

42. Concordia. [http://concordia.csail.mit.edu].

43. Bodenreider O: The Unified Medical Language System (UMLS): integrating biomedical terminology. Nucleic Acids Res 2004, 32:D267-270.

44. Osborne JD, Lin S, Zhu L, Kibbe WA: Mining biomedical data using MetaMap Transfer (MMtx) and the Unified Medical Language System (UMLS). Methods Mol Biol 2007, 408:153-169.

45. Affymetrix: Affymetrix Microarray Suite User Guide. [http://www.affymetrix. com/support/technical/manuals.affx].
46. R Development Core Team: R: A Language and Environment for Statistical Computing. Vienna, Austria 2007.

47. Gentleman RC, Carey VJ, Bates DM, Bolstad B, Dettling M, Dudoit S, Ellis B, Gautier L, Ge Y, Gentry J, Hornik K, Hothorn T, Huber W, lacus S, Irizarry R, Leisch F, Li C, Maechler M, Rossini AJ, Sawitzki G, Smith C, Smyth G, Tierney L, Yang JYH, Zhang J: Bioconductor: open software development for computational biology and bioinformatics. Genome Biol 2004, 5:R80.

48. Kohane IS, Butte AJ, Kho A: Microarrays for an Integrative Genomics Cambridge, MA, USA: MIT Press; 2002

49. Falcon S, Gentleman R: Using GOstats to test gene lists for GO term association. Bioinformatics 2007, 23:257-258.

doi:10.1186/gb-2012-13-8-r71

Cite this article as: Palmer et al: A gene expression profile of stem cell pluripotentiality and differentiation is conserved across diverse solid and hematopoietic cancers. Genome Biology 2012 13:R71.

\section{Submit your next manuscript to BioMed Central and take full advantage of:}

- Convenient online submission

- Thorough peer review

- No space constraints or color figure charges

- Immediate publication on acceptance

- Inclusion in PubMed, CAS, Scopus and Google Scholar

- Research which is freely available for redistribution

Submit your manuscript at www.biomedcentral.com/submit
Biomed Central 